\title{
Is Routine Uroflowmetry Necessary following Hypospadias Repair?
}

\author{
Caroline Smith* and Prasad Godbole \\ Department of Paediatric Urology, Sheffield Children's Hospital, UK
}

*Corresponding author: Caroline Smith, Department of Paediatric Urology, Sheffield Children's Hospital, Western Bank, Sheffield, S102TH, UK, E-mail: c.m.smith@doctors.org.uk

\begin{abstract}
Summary
Introduction: Uroflow parameters post hypospadias repair are known to remain poor initially but most improve with time. There is a recognised group of patients who develop late deterioration of flow and may require surgical intervention, but at present there are no standardised guidelines as to length and nature of follow up.
\end{abstract}

Objective: We aim to analyse whether routine uroflow studies at 5 years of age are predictive of need for delayed surgery in children with and without voiding symptoms.

Study design: A retrospective review of all children having undergone hypospadias repair at a single institute in a single year (2000) with routine uroflow at 5 years and follow up where necessary into puberty. Data analysis was with Fishers exact.

Results: Seventy-two patients were identified who had undergone hypospadias repair and completed uroflowmetry assessment at 5 years of age. The population reflected the standard UK distribution of grade of hypospadias defect.

$26 \%$ of children had a normal uroflow study at 5 years. Abnormal uroflow rates (Qmax) predicted need for surgery ( $p$ $=0.01$ ).

Twenty children were symptomatic. All needed surgical intervention (OR 36 (95\% Cl 8.5-151.5)) except two children with normal flow rates.

In fifty-two asymptomatic children, 69\% had low Qmax on uroflow and underwent annual follow up. Of these four children required subsequent surgery around puberty. No asymptomatic child with normal uroflow findings at 5 years required subsequent surgery.

Discussion: This study highlights the merits of a routine uroflow at the age of 5 years. Abnormal uroflow parameters predicts need for late surgery. Uroflow is particularly useful in two groups of children: In asymptomatic patients with normal uroflow parameters it allows the clinician to confidently discharge the patient and in those with subjective symptomology identifies those with normal voiding mechanics, avoiding unnecessary surgery.
Conclusion: We found routine uroflow at age 5 years following hypospadias is predictive of the need for regular follow up and potential for late deterioration needing surgery. We suggest a follow up algorithm based on uroflow findings at age 5 years in combination with voiding symptomology, as shown in Table 1.

\section{Keywords}

Hypospadias, Uroflow, Long term follow up

Abbreviations
Qmax: Maximum urinary flow rate; OR: Odd Ratio; CI: Con-
fidence Interval

\section{Introduction}

The aim of hypospadias repair is to improve functionality for standing micturition and fecundity. It is widely recognised that following surgery urinary flow rarely returns to 'normal' [1,2]. Even where cosmetic results are good and the surgery is complication free, uroflow findings a year post repair show at least half of boys have a flow rate (Qmax) under the $5^{\text {th }}$ centile for age, with classic plateaux shaped curves [3-5]. This improves with time in many children [3], but a proportion continue to have poor uroflow parameters into puberty. Adult studies show approximately $11-14 \%$ of patients with previously repaired hypospadias continue with poor urinary flow rates $[6,7]$, and $5-10 \%$ of boys will require late surgical intervention for voiding dysfunction $[8,9]$.

At present there is no clear evidence that poor uroflow parameters are predictive of voiding deterioration and requirements for late surgery. At our unit we perform a routine uroflow in all children having undergone previous hypospadias repair at the age of 5 years. As-

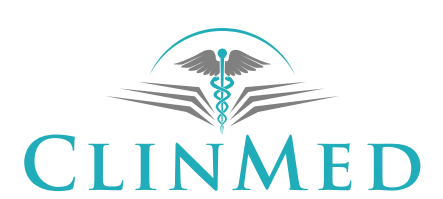

INTERNATIONAL LIBRARY

Citation: Smith C, Godbole P (2018) Is Routine Uroflowmetry Necessary following Hypospadias Repair?. Int Arch Urol Complic 4:038. doi.org/10.23937/2469-5742/1510038

Accepted: May 23, 2018: Published: May 25, 2018

Copyright: (c) 2018 Smith C, et al. This is an open-access article distributed under the terms of the Creative Commons Attribution License, which permits unrestricted use, distribution, and reproduction in any medium, provided the original author and source are credited. 
Table 1: Proposed follow up protocol at 5 years of age with uroflow results following hypospadias repair. NB. Abnormal uroflow findings include low Qmax, prolonged micturition time, significant residuals or plateaux shaped curve.

\begin{tabular}{|l|l|l|}
\hline \multicolumn{1}{|c|}{ Abnormal Voiding Symptoms } \\
\hline Uroflow findings & Yes & No \\
\hline Normal Uroflow Parameters & $\begin{array}{l}\text { Can be discharged if stable with no deterioration, with advice to return } \\
\text { if worsening voiding patterns }\end{array}$ & Can be discharged \\
\hline Abnormal Uroflow Parameters & Annual Review & Annual Review \\
\hline
\end{tabular}

ymptomatic boys with normal uroflows are discharged. Those with symptoms and abnormal uroflows are seen with a view to surgical intervention. Boys who remain asymptomatic but with abnormal low uroflows are reviewed annually with uroflowmetry up to the age of puberty. In this study we aim to assess whether uroflow results at age 5 years, in addition to clinical symptoms, are predictive of those children requiring late surgical intervention (after 5 years of age) following hypospadias repair.

\section{Materials and Methods}

All children undergoing hypospadias repair at a single centre in a single year (2000) were identified from a specialist paediatric urology database. Children were included in analysis if they had completed at least 5 years follow up with uroflow results available and follow up into puberty where deemed necessary.

Children underwent primary procedure between the ages of 9 and 12 months. At our institute conservative management is pursued for glanular hypospadias; meatal advancement for very distal hypospadias; tubularisation with or without posterior plate incision for the majority of distal, mid and some severe hypospadias; and two-stage for penoscrotal and proximal hypospadias. All children were seen a year post hypospadias repair and underwent routine uroflow analysis at 5 years of age. Children who were symptomatic were followed up more intensely as appropriate.

Data collection included patient demographics, post-operative symptomology and uroflow parameters at routine uroflow at 5 years post repair. These parameters included flow rates and post void residual bladder volumes. The primary outcome measured was need for late surgical intervention (after 5 years of age). The outcomes were compared by uroflow findings and by presence or absence of symptoms. Fishers exact was used for nominal data analysis, Mann Whitney $U$ test for non-parametric continuous.

\section{Results}

Seventy-two patients were identified who had undergone hypospadias repair and completed a minimum of 5 years follow up with or without follow up into puberty, with available uroflow results. The population reflected the standard UK distribution of grade of hypospadias defect.

Only $26 \%$ of children had a normal uroflow study at 5 years, with 74\% showing low Qmax, plateaux shaped curve, residual volumes $>10 \%$ and in some interrupted flow patterns. Of those with poor flow rates at age 5 years $44 \%$ required surgery, uroflow predicted need for surgery $(p=0.01)$. Overall $26 \%$ of children required surgical intervention for complications following hypospadias repair.

Twenty children were symptomatic at 5 years, of whom $75 \%$ underwent surgery. Voiding symptomology at 5 years indicated a need for surgical intervention (OR 36 (95\% Cl 8.5-151.5)), except in two children who had normal flow rates despite described voiding problems; who did not progress to surgery.

In fifty-two asymptomatic children, 69\% had low Qmax on uroflow analysis at 5 years. Of these asymptomatic children four children required subsequent surgery. Abnormal uroflow findings significantly increased your risk of requirements for surgery (OR $9.25(95 \% \mathrm{Cl} 1.14-75.0))$, highlighting those children with subclinical urinary flow dysfunction. Urinary flow rate was the primary uroflow parameter predictor of outcomes, irrespective of absolute or relative post void residual volumes.

No asymptomatic child with normal uroflow findings at 5 years required subsequent surgery, with a negative predictive value of $100 \%$.

The age of surgical intervention in this population was between 5 years and 16 years. Cases presenting later showed urethral diverticula and stenosis rather than fistulae, which tended to present in the early post-operative phase, see Figure 1.

\section{Discussion}

Complications post hypospadias repair are well described, with approximately $20 \%$ of all comers requiring further surgery [10]. Approximately half will require a further operation within year one, 38\% after year two and $5 \%$ after year three [8]. It is of interest that severity of hypospadias and method of repair does not seem to correlate with post-operative uroflow findings $[4,7,10]$, and neither does repaired urethral size $[1,2]$. This had led to speculation, with support from modelling experiments, that it is poor compliance of the neourethra, due to its position within the stiffened tissues of healing glans that slows urinary flow $[4,5,11]$.

With no consensus on follow up following hypospadias repair, there is a risk that units discharging children with a single normal uroflow following potty training will miss young adults with evolving voiding dysfunction due to the changes in hormone stimulation of the tis- 

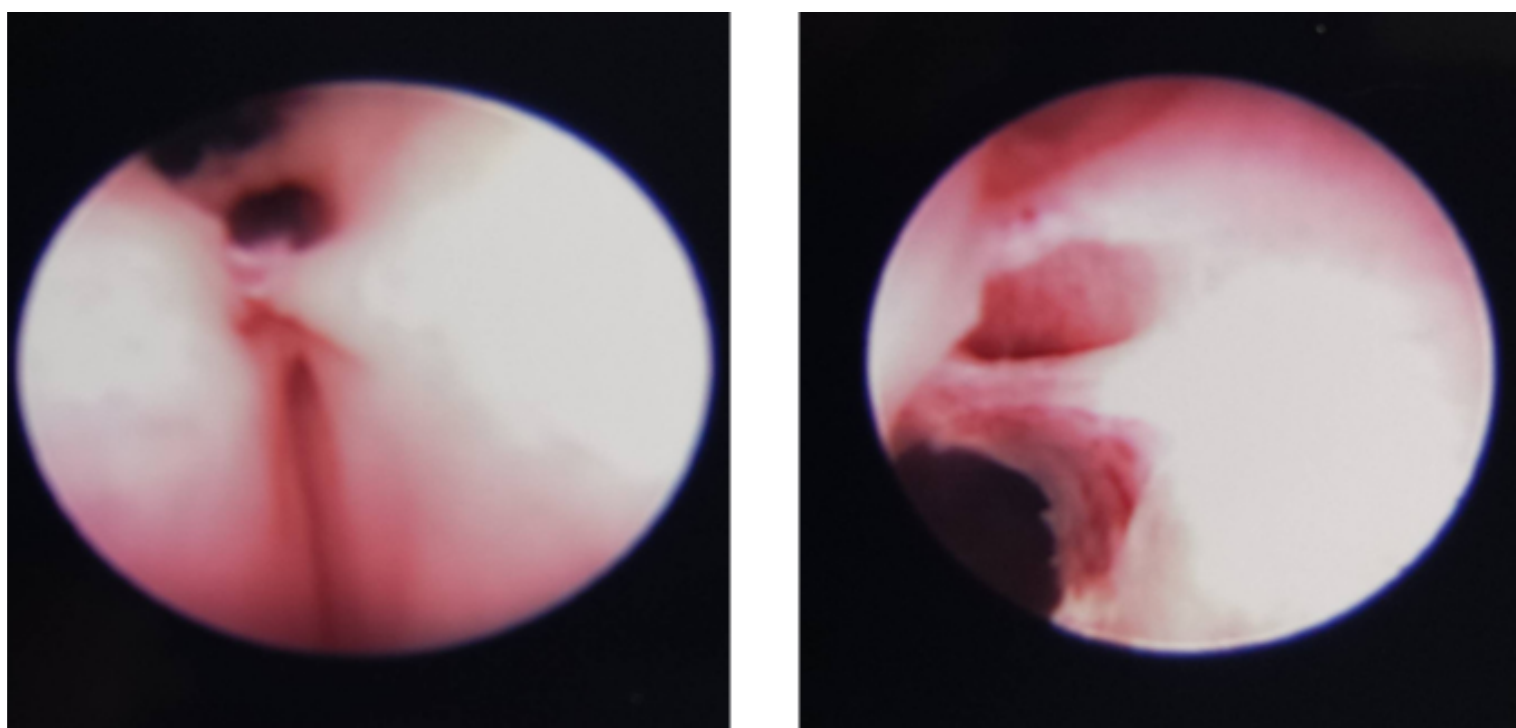

Figure 1: Showing endoscopic findings diverticula flap and neourethra in 2 boys following hypospadias repair: a) A 15-year-old following distal repair; b) A 16-year-old following a 2 stage repair.

sues around the time of puberty [3]. Previous reports have suggested one uroflow post potty training is sufficient [10] whereas other papers have supported undefined long term follow up [8]. In addition, the sexual and psychological impact of infant penile surgery on the pubescent male should not be forgotten $[12,13]$, and therefore routine appointment at puberty offers the opportunity for questions and reassurance.

We recommend routine uroflow at 5 years of age and have shown distinct advantages in 2 groups. The first is the child with 'normal' experience of micturition. An abnormal uroflow may predict deteriorating voiding patterns and allow early intervention with urotherapy and bladder training to prevent later surgery or allow earlier surgical intervention. The second is in the group with symptomatic voiding patterns where the subjective experience is at odds with the objective uroflow findings. In our cohort uroflow prevented unnecessary intervention in $10 \%$ of subjectively 'symptomatic' voiders.

\section{Conclusion}

There are an appreciable number of children who require delayed surgical intervention post hypospadias repair. In particular our findings indicate puberty is a time where mild symptomology or poor flow rates are provoked into necessitating late surgical intervention. From this study we would make recommendations for the long term follow up of children following hypospadias repair as shown in Table 1. Where a child is asymptomatic with a normal uroflow at 5 years they are unlikely to require future surgery and can be discharged from follow up. Where a child is asymptomatic but has low flow rates, we would recommend annual review. Where is a child is symptomatic we would recommend annual review but in the case of a normal uroflow if symptoms do not worsen this child may be discharged with advice to return if symptoms worsen.

\section{Conflict of Interest Statement}

We declare no conflict of interest.

\section{Acknowledgements}

The urology nurse team at Sheffield Children's Hospital: Jo Searles, Carol Hopkiss and Sarah Boulby.

\section{Ethical Approval}

Study design was approved with Sheffield Children's Hospital Clinical Governance department.

\section{References}

1. Page RE, Akin Y (1978) Assessment of urine flow in hypospadias. Br J Plast Surg 31: 313-316.

2. Olsen LH, Grothe I, Rawashdeh YF, Jørgensen TM (2011) Urinary flow patterns in infants with distal hypospadias. J Pediatr Urol 7: 428-432.

3. Andersson M, Doroszkiewicz M, Arfwidsson C, Abrahamsson K, Holmdahl G (2011) Hypospadias repair with tubularized incised plate: Does the obstructive flow pattern resolve spontaneously? J Pediatr Urol 7: 441-445.

4. Malyon AD, Boorman JG, Bowley N (1997) Urinary flow rates in hypospadias. Br J Plast Surg 50: 530-535.

5. Wolffenbuttel KP, Wondergem N, Hoefnagels JJ, Dieleman GC, Pel JJ, et al. (2006) Abnormal urine flow in boys with distal hypospadias before and after correction. J Urol 176: 1733-1736.

6. Robinson AJ, Harry LE, Stevenson JH (2013) Assessment of long term function following hypospadias reconstruction: do flow rates, flow quality and cosmesis improve with time? Results from the modified Bretteville technique. Journal of Plastic, Reconstructive \& Aesthetic Surgery 66: 120-125.

7. Perera M, Jones B, O'Brien M, Hutson JM (2012) Longterm urethral function measured by uroflowmetry after hypospadias surgery: Comparison with an age matched control. J Urol 188: 1457-1462.

8. Spinoit A-F, Poelaert F, Groen L-A, Van Laecke E, Hoebeke $P$ (2013) Hypospadias repair at a tertiary care center: Long-term followup is mandatory to determine the real complication rate. J Urol 189: 2276-2281. 
9. Goyal A, Singh MV, Dickson AP (2010) Oral mucosa graft for repair of hypospadias: Outcomes at puberty. J Urol 184: 2504-2508.

10. González R, Ludwikowski BM (2011) Importance of urinary flow studies after hypospadias repair: A systematic review. Int J Urol 18: 757-761.

11. Idzenga T, Kok DJ, Pel JJ, van Mastrigt R, Wolffenbuttel KP (2006) Is the impaired flow after hypospadias correction due to increased urethral stiffness? J Pediatr Urol 2: 299-303.

12. Hoag CC, Gotto GT, Morrison KB, Coleman GU, Macneily $A E$ (2008) Long-term functional outcome and satisfaction of patients with hypospadias repaired in childhood. Can Urol Assoc J 2: 23-31.

13. Aulagne MB, Harper L, de Napoli-Cocci S, Bondonny JM, Dobremez E (2010) Long-term outcome of severe hypospadias. J Pediatr Urol 6: 469-472. 